\title{
Variables perinatales de recién nacidos de madres Aymara sugieren adaptación genética a la altura
}

\author{
MARÍA PIZARRO-ORTIZ ${ }^{1,2, a}$, RODRIGO BARRA ${ }^{3}$, \\ FELIPE GAJARDO ${ }^{4}$, MACARENA FUENTES-GUAJARDO ${ }^{1,2, a}$, \\ FRANCISCO ROTHHAMMER ${ }^{1,2,3, b}$

\section{Perinatal variables from newborns of Aymara mothers suggest a genetic adaptation to high altitude}

Background: Studies performed in Andean populations living in high altitude, indicate that the reduced availability of oxygen could be associated to both a fetal growth retardation and a lower birth weight. These variables are predictive of morbidity and mortality during the first year of life. Aim: To study perinatal variables of newborns of mothers living at contrasting altitudinal levels, harboring different degrees of Aymara ancestry. Subjects and Methods: Review of medical records of 5,295 women whose deliveries occurred between February 2004 and August 2010. Information was obtained on place of residence, grouped into two categories: coast (150 to 3,000 m) and high plateau (3,000 to 4,300 m), ancestry was estimated using number of Aymara surnames that were homologated to percentages of Amerindian admixture, gestational age, birth weight, height, head circumference and obstetric variables. Results: Gestational age showed a tendency to increase and birth weight, height and head circumference to decrease with altitude of residence. Only weight reached statistical significance. Women with Aymara ancestry gave birth to children with a significantly higher gestational age, weight and cranial circumference. Conclusions: Altitude of residence is related to a decrease in perinatal variables that proved to be less pronounced in newborns of mothers with a higher degree of Aymara ancestry. Results suggest a genetic adaptation to hypoxia that could be related to candidate genes linked to the capture, transport or utilization of oxygen.

(Rev Med Chile 2014; 142: 961-965)

Key words: Attitude; Environment; Indians, South American; Infant, newborn.

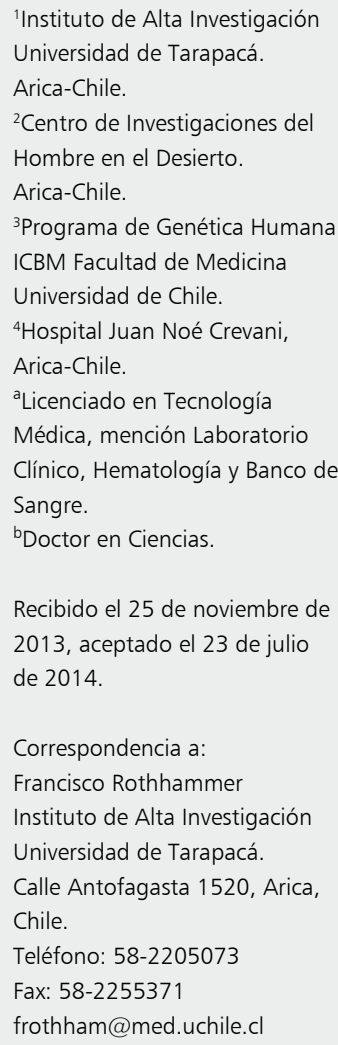

L os ecosistemas de altura se caracterizan por una disminución de la presión parcial de oxígeno asociada a su vez a una menor presión barométrica, que genera como consecuencia un déficit de oxígeno a nivel tisular ${ }^{1,2,3}$. Estudios realizados en poblaciones originarias de los Andes indican que la menor disponibilidad de oxígeno estaría asociada tanto a un retardo del crecimiento fetal como a un menor peso de nacimiento, varia- ble considerada predictiva de morbilidad y mortalidad infantil durante el primer año de vida ${ }^{1,4}$. En el presente trabajo se analiza la distribución de un conjunto de variables perinatales de recién nacidos (RN) de la Región de Arica-Parinacota, que se diferencian principalmente en cuanto a la altura del lugar de residencia y la ancestría aymara de las madres, con el objetivo de investigar si existe en el extremo norte de Chile una relación entre 
estas variables. Con esta finalidad se recurrió al universo completo de registros administrativos del Hospital Juan Noé, consignándose la altura de residencia de las madres de origen aymara y algunas variables perinatales especialmente el peso y talla de nacimiento que son utilizadas para evaluar el crecimiento y estado nutricional en recién nacidos $(\mathrm{RN})^{4}$.

\section{Materiales y Métodos}

El presente estudio es de tipo observacional, transversal, descriptivo y comparativo, con un muestreo no probabilístico y de conveniencia, de acuerdo a los criterios de inclusión y exclusión. La información fue recolectada del libro de partos del Hospital Juan Noé Crevani de Arica, registrándose el universo completo de nacimientos ocurridos entre febrero de 2004 y agosto de 2010. Los datos de previsión social, lugar de residencia, edad de la madre, antecedentes obstétricos, morbilidad materna asociada al embarazo y datos neonatales incluyendo género, edad gestacional, talla, peso y circunferencia craneana de los RN fueron obtenidos de las fichas clínicas del hospital. Se incluyeron los antecedentes de aquellas madres cuyos partos fueron atendidos entre las 37 y 40 semanas de gestación, cuyas edades estuvieron entre los 15 y 40 años y dieron a luz $\mathrm{RN}$ vivos, sanos, únicos y que presentaban mediciones perinatales. Con la finalidad de no introducir factores de sesgo en el análisis de asociación, se excluyeron de este estudio los RN de madres con embarazos múltiples, antecedentes de consumo de sustancias psicoactivas, tabaquismo, alcoholismo y patologías que pudiesen modificar de alguna forma las variables perinatales. No fueron consideradas fichas clínicas con información incompleta. La población estudiada comprendió 5.295 madres.

Los RN se clasificaron de acuerdo a género, edad materna, edad gestacional, nivel socioeconómico, altitud de residencia y ascendencia aymara. Las madres se agruparon en adolescentes (menores de 20 años), jóvenes ( 20 a 27 años), adultas jóvenes (28-39 años) y adultas (sobre 40 años). El nivel socioeconómico (NSE) fue obtenido a través de la ubicación en las categorías del Fondo Nacional de Salud (FONASA). Los antecedentes del lugar de residencia se obtuvieron de las fichas clínicas. Estos fueron clasificados de acuerdo a la altura geográfi- ca, siendo separados en dos zonas geoecológicas, costa $(<3.000$ m.s.n.m.), y altiplano (> 3.000 m.s.n.m.) debido a que el peso de nacimiento disminuye sobre 3.000 m.s.n.m. (ver Resultados). Estas zonas, como es sabido, difieren en numerosas características entre las cuales sobresalen la baja presión atmosférica, la gran amplitud térmica diaria, la escasa humedad y la alta radiación solar y cósmica ${ }^{1}$. La ancestría aymara se determinó utilizando el número de apellidos de origen aymara. Datos previos obtenidos por nuestro grupo con base en 40 polimorfismos de secuencia única indicadores de ancestría (AIM's), indican que los individuos sin apellidos aymara de la Región de Arica-Parinacota resultaron tener un componente genético amerindio de $51,12 \pm 20,09 \%$, con un apellido aymara de $68,33 \% \pm 16,79 \%$ y con dos apellidos aymara de $99,68 \% \pm 0,49 \%{ }^{5}$. Los porcentajes de mezcla difieren significativamente ( $\mathrm{p}>0,0001)$.

Los datos obtenidos de las fichas clínicas fueron ingresados al programa estadístico SPSS versión 20.0 para Windows. Se comprobó la normalidad de las distribuciones con las pruebas de Kolmogorov-Smirnov (con la corrección de Lilliefors) y Shapiro-Wilk y la homogeneidad de varianzas a través de la prueba de Levene. La prueba de ANOVA de un factor permitió obtener la significación estadística de la variancia de edad gestacional, peso, talla y circunferencia craneana atribuible a lugar de residencia y ancestría aymara. Para evaluar el efecto independiente de estas variables se utilizó la prueba de Student.

Esta investigación fue aprobada por el Comité Ético Científico del Ministerio de Salud para la zona norte, con sede en Coquimbo, el 20 de julio de 2007 y posteriormente ratificado por el Comité de Bioética del Servicio de Salud de la Región de Arica y Parinacota y por el Comité de Bioética del Hospital Dr. Juan Noé Crevani, enmarcándose dentro de las normas bioéticas internacionales de investigación en seres humanos.

\section{Resultados}

Del total de 5.295 RN, 2.621 correspondieron a mujeres, 2.672 a varones y dos no presentaban información sobre género. El número de $\mathrm{RN}$ de ambos géneros no difiere significativamente. Los varones presentaron pesos de nacimiento, tallas 
y medidas de circunferencia craneana levemente mayores. Las diferencias alcanzan en todos los casos significación estadística. Las variables neonatales variaron significativamente con la edad materna, presentando una distribución caracterizada por valores más bajos en las madres adolescentes y jóvenes ( $>27$ años) y adultas $(>40$ años) y valores levemente más altos entre los 28 y 39 años. El 89,36\% de las madres pertenecía a los NSE más bajos (Fonasa A, B y Sin Previsión), el remanente a los más altos (Fonasa C, D y Particular). Con el propósito de verificar si el NSE influye en los resultados del presente estudio, se dividieron los grupos de bajos y altos ingresos según altura de residencia, poniendo como límite los 3.000 m.s.n.m. No se detectó una diferencia estadísticamente significativa para NSE entre los grupos ( $\mathrm{p}>0,1985)$.

$\mathrm{Al}$ considerar el efecto de altura de residencia, los promedios de edad gestacional con desviaciones estándar entre paréntesis en semanas fueron $39,1059(0,97)$ (costa) y 39,1944 $(1,05)$ (altiplano); de peso al nacer en gramos fueron $3.452,66$ $(426,37)$ (costa) y $3.295,74(391,56)$ (altiplano); de talla en cm fueron 49,95 $(1,88)$ (costa) y 49,64 $(1,77)$ (altiplano) y por último, de perímetro craneano en $\mathrm{cm}$ fueron $34,76(1,28)$ (costa) y 34,68 $(1,09)$ (altiplano). El ANOVA entre las variables analizadas y la altura de residencia demostró que solamente el peso presentó una disminución estadísticamente significativa $(\mathrm{p}<0,011)$. Luego, analizando los promedios de peso entre tres alturas geográficas costa $(<2.000$ m.s.n.m.), sierra $(2.000$ y 3.000 m.s.n.m.) y altiplano (> 3.000 m.s.n.m) se observaron diferencias significativas entre costa y altiplano $(\mathrm{p}=0,019)$ y sierra y altiplano $(\mathrm{p}=0,017)$. En consecuencia, altura de residencia disminuyó el peso de nacimiento sobre 3.000 m.s.n.m.

$\mathrm{Al}$ analizar ancestría aymara en relación con las variables dependientes, el ANOVA resultante demostró un aumento significativo de edad gestacional $(\mathrm{p}<0,002)$, peso de nacimiento $(\mathrm{p}<0,006)$, talla $(\mathrm{p}<0,000)$ y perímetro craneano $(\mathrm{p}<0,028)$.

Considerando que la altura afecta significativamente al peso de nacimiento sobre los $3.000 \mathrm{~m}$, la evaluación del efecto independiente de lugar de residencia y ancestría aymara se realizó considerando dos niveles de altitud, costa $(<3.000$ m.s.n.m.) y altiplano (> 3.000 m.s.n.m.) y dos niveles de ancestría, sin y con apellidos aymara. Homologando el número de apellidos a los índi- ces de mezcla genética obtenidos previamente, el grupo sin apellidos aymara tiene un valor esperado de cerca de $50 \%$ de mezcla aymara y el grupo con apellidos aymara cerca de $80 \%$. Al inspeccionar la Tabla 1, se aprecia que a nivel de costa los RN de madres con apellidos aymara exhiben valores mayores, altamente significativos, para las cuatro variables perinatales estudiadas. La misma tendencia se observa a nivel de altiplano, resultando sólo significativas las diferencias de estatura y edad gestacional, probablemente debido al pequeño tamaño de algunas muestras.

Los RN de madres sin apellidos aymara presentaron una reducción significativa de peso en altura, siguiendo una tendencia esperada. Los RN de madres con apellidos aymara presentaron una reducción menor y contrariamente a los $\mathrm{RN}$ de madres sin ancestría aymara, exhibían un aumento significativo de la edad de gestación. Al comparar RN nacidos en la costa con nacidos en el altiplano, los últimos presentaron un valor significativamente más bajo de peso al nacer. Al comparar a su vez RN de madres con y sin apellidos aymara, las cuatro variables perinatales estudiadas presentaron valores significativamente más altos en $\mathrm{RN}$ de madres con apellidos aymara. Resumiendo, la exposición a la altura se tradujo en un aumento de la edad de gestación para el grupo con mayor ancestría aymara y una disminución del peso, de la talla y de la circunferencia craneana en ambos grupos, siendo esta menor en el grupo predominantemente aymara. Destacamos además el hecho que este mismo grupo presentó a nivel de la costa valores significativamente más altos para todas las variables dependientes analizadas (Tabla 1).

\section{Discusión}

Los primeros americanos fueron capaces de adaptarse a una variedad de entornos más o menos rigurosos ${ }^{6}$. Una de las características consistentemente relacionadas con la adaptación a la altura es el menor peso y el reducido tamaño corporal de los RN de mujeres residentes ${ }^{7,8}$. Nuestros resultados respaldan esta observación. Los RN de madres que viven en el altiplano chileno pesan menos $(3.295,74 \mathrm{~g})$ en comparación con los RN de madres residentes en la costa $(3.453,65 \mathrm{~g})$. Si bien esta diferencia es altamente significativa, ambos valores se encuentran entre el percentil 50 
Adaptación genética a la altura en recién nacidos Aymaras - M. Pizarro-Ortiz et al

Tabla 1. Promedios con desviaciones estándar de variables perinatales en relación a altura de residencia y ancestría aymara de las madres

\begin{tabular}{|c|c|c|c|c|c|c|}
\hline \multicolumn{2}{|c|}{$\begin{array}{l}\text { Residencia/apellido } \\
\text { Aymara }\end{array}$} & \multirow{2}{*}{$\begin{array}{c}\mathbf{n} \\
4,005\end{array}$} & \multirow{2}{*}{$\begin{array}{c}\begin{array}{c}\text { Peso } \\
\text { (kg) }\end{array} \\
3,44(0,43)\end{array}$} & \multirow{2}{*}{$\begin{array}{c}\begin{array}{c}\text { Estatura } \\
(\mathrm{cm})\end{array} \\
49,56(1,87)\end{array}$} & \multirow{2}{*}{$\begin{array}{c}\text { Circunferencia } \\
\text { craneana }(\mathrm{cm}) \\
34,73(1,29)\end{array}$} & \multirow{2}{*}{$\begin{array}{c}\text { Edad gestacional } \\
\text { (sem) } \\
39,08(0,97)\end{array}$} \\
\hline Costa & No & & & & & \\
\hline & Sí & 1,236 & $3,49(0,43)$ & $50,26(1,87)$ & $34,84(1,27)$ & $39,19(0,99)$ \\
\hline & $p<$ & & 0,0005 & 0,0005 & 0,005 & 0,0005 \\
\hline \multirow[t]{3}{*}{ Altiplano } & No & 8 & $3,26(0,38)$ & $49,00(2,00)$ & $34,31(0,96)$ & $38,63(0,78)$ \\
\hline & Sí & 46 & $3,30(0,40)$ & $49,75(1,72)$ & $34,75(1,11)$ & $39,23(1,07)$ \\
\hline & $p<$ & & 0,400 & 0,0005 & 0,200 & 0,050 \\
\hline \multirow[t]{3}{*}{ No } & Costa & 4,005 & $3,44(0,43)$ & $49,56(1,87)$ & $34,73(1,29)$ & $39,08(0,97)$ \\
\hline & Altiplano & 8 & $3,26(0,38)$ & $49,00(2,00)$ & $34,31(0,96)$ & $38,63(0,78)$ \\
\hline & $p<$ & & 0,0005 & 0,100 & 0,200 & 0,100 \\
\hline \multirow[t]{3}{*}{ Sí } & Costa & 1,236 & $3,49(0,43)$ & $50,26(1,87)$ & $34,84(1,27)$ & $39,19(0,99)$ \\
\hline & Altiplano & 46 & $3,30(0,40)$ & $49,75(1,72)$ & $34,75(1,11)$ & $39,23(1,07)$ \\
\hline & $p<$ & & 0,005 & 0,0005 & 0,350 & 0,0005 \\
\hline \multicolumn{2}{|c|}{ Total Costa } & 5,241 & $3,45(0,43)$ & $49,96(1,88)$ & $34,76(1,29)$ & $39,11(0,97)$ \\
\hline \multirow{2}{*}{\multicolumn{2}{|c|}{ Total Altiplano }} & 54 & $3,30(0,39)$ & $49,64(1,77)$ & $34,68(1,10)$ & $39,19(1,06)$ \\
\hline & & & 0,007 & 0,217 & 0,669 & 0,504 \\
\hline \multicolumn{2}{|c|}{ Total no-Aymara } & 4,013 & $3,44(0,43)$ & $49,86(1,87)$ & $34,73(1,29)$ & $39,08(0,97)$ \\
\hline \multicolumn{2}{|c|}{ Total Aymara } & 1,282 & $3.49(0,43)$ & $50,24(1,87)$ & $34,84(1,27)$ & $39,19(0,99)$ \\
\hline & $p<$ & & 0,001 & $<0,001$ & 0,008 & $<0,001$ \\
\hline
\end{tabular}

Altura: Costa y Altiplano. Con Apellido Aymara: Sí; Sin Apellido Aymara: No. Comparaciones significativas en negrita.

y 75 de las curvas de Alarcón-Pittaluga ${ }^{9}$ para peso al nacer de neonatos chilenos recomendadas por la Sociedad de Pediatría de Chile. Al comparar el promedio de peso de $\mathrm{RN}$ residentes en el altiplano chileno con el peso de RN residentes en otros lugares que se encuentran sobre los $3.000 \mathrm{~m}$ de altura como La Paz (Bolivia) $(3.131 \mathrm{~g})^{10}$, Susques (Argentina) $3.145,80 \mathrm{~g}^{11}$, Antofagasta de la Sierra (Argentina) 3.037,52 g ${ }^{12}$, Colorado (Estados Unidos de Norteamérica) $3.126 \mathrm{~g}^{13}$ y Cusco (Perú) $3.262 \mathrm{~g}^{14}$ se observan valores bastante semejantes, quizás con excepción de Antofagasta de la Sierra en que la maternidad temprana junto a las precarias condiciones socioeconómicas propias de la zona, podrían incidir significativamente ${ }^{12}$.

Esta investigación demuestra que los $\mathrm{RN}$ de mujeres con mayor ancestría aymara presentan valores más altos para las medidas perinatales en comparación con los RN de mujeres con menor ancestría aymara. Estos resultados concuerdan con investigaciones realizados en Perú y Bolivia, que señalan que las mujeres descendientes de familias con más de tres generaciones de vida en altura, presentan una mejor saturación arterial de oxígeno y los RN tienen un mejor peso de nacimiento en comparación con aquellas que han vivido menos tiempo en altura ${ }^{7,15,16}$. Estudios en que se mide el flujo de sangre y el transporte de oxígeno en mujeres residentes en altura con ascendencia andina en comparación con mujeres con ascendencia europea, señalan que las primeras tienen una mayor capacidad de aumentar el intercambio de oxígeno durante el embarazo y mejor flujo sanguíneo que las segundas ${ }^{17}$. Estos resultados posiblemente sean consecuencia de adaptaciones fisiológicas que ocurren durante la vida en altura. Sin embargo, el hallazgo que los $\mathrm{RN}$ de madres con uno o dos apellidos aymaras tengan un peso significativamente mayor a las madres sin apellidos aymara a nivel de la costa 
Adaptación genética a la altura en recién nacidos Aymaras - M. Pizarro-Ortiz et al

hace pensar más bien en una adaptación genética debido a que esta diferencia no se puede explicar por diferencias en NSE (ver Resultados). Es posible que, considerando la historia de residencia en altura y además el hecho que la hipoxia afecta fundamentalmente al feto, se hayan seleccionado en las poblaciones andinas genes relacionados con la captación fetal de oxígeno ${ }^{18}$.

\section{Conclusiones}

La Región de Arica-Parinacota se caracteriza por presentar marcadas diferencias altitudinales, pudiendo la hipoxia provocar una disminución del peso de nacimiento que es un importante indicador de morbilidad y mortalidad infantil durante el primer año de vida. Hemos podido demostrar que el poseer una mayor ancestría aymara se relaciona con una disminución menos acentuada de esta variable perinatal, confiriendo posiblemente una protección contra los efectos negativos de la hipoxia. En consecuencia, resulta razonable suponer que durante las 400 a 500 generaciones que los ancestros de las poblaciones andinas han habitado ecosistemas de altura, se hayan seleccionado genes que contribuyen a determinar la protección descrita y que eventualmente podrían ser identificados utilizando técnicas de biología molecular.

Agradecimientos: Agradecemos al equipo de matronas del Hospital Dr. Juan Noé, región de Arica y Parinacota, Chile por su entusiasta y desinteresada participación en el presente estudio. Asimismo al Editor y a los revisores que contribuyeron a mejorar este artículo.

\section{Referencias}

1. Schull WJ. and Rothhammer F. The Aymará: Strategies in human adaptation to a Rigorous Environment. Kluwer Academic Publishers. Boston 1990.

2. Hammond KA, Szewczak J, Król E. Effects of altitude and temperature on organ phenotypic plasticity along an altitudinal gradient. J Exp Biol 2001; 204: 1991-2000.

3. Storz JF, Scott GR, Cheviron Z. Phenotypic plasticity and genetic adaptation to high-altitude hypoxia in vertebrates. J Exp Biol 2010; 213: 4125-36.
4. Cárdenas-López C, Haua-Navarro K, Suverza-Fernández A, Perichart-Perera O. Mediciones antropométricas en el neonato. Bol Med Hosp Infant Mex 2005; 62: 214 24.

5. Fuentes M, Pulgar I, Gallo C, Bortolini M-C, CanizalesQuinteros S, Bedoya G, et al. Geografía génica de Chile. Distribución regional de los aportes genéticos americanos, europeos y africanos. Rev Med Chile 2014; 142: 281-9.

6. Rothhammer F, Dillehay T. The late Pleistocene colonization of South America: an interdisciplinary perspective. Ann Hum Genet 2009; 70: 540-9.

7. Bennett A, Sain SR, Vargas E, Moore LG. Evidence that parent-of-origin affects birth-weight reductions at high altitude. Am J Hum Biol 2008; 20: 592-7.

8. Julian CG. High altitude during pregnancy. Clin Chest Med 2011; 32: 21-31.

9. Milad M, Novoa JM, Fabres J, Samamé M, Aspillaga C. (2010) Recomendación sobre Curvas de Crecimiento Intrauterino. Rev Chil Pediatr 2010; 81 (3): 264-74.

10. Mazzi E. Antropometría neonatal Rev. Soc. Boliv. Pedia$\operatorname{tr} 1985 ; 24:$ 43-55.

11. Lomaglio D, Marrodán M, Verón J, Díaz M, Gallardo F, Alba J, et al. Peso al nacimiento en comunidades de altura de la Puna Argentina: Antofagasta de la Sierra (Catamarca). Antropol 2005; 9: 61-70.

12. Moreno S, Marrodán M, Dipierri J. Peso de nacimiento en ecosistemas de altura. Noroeste argentino: Susques. Observ Medioamb 2003; 6: 161-76.

13. Hartinger S, Tapia V, Carrillo C, Bejarano L, Gonzales GF. Birth weight at high altitudes in Peru. Int J Gynaecol Obstet 2006; 93: 275-81.

14. McAuliffe F, Kametas N, Krampl E, Ernsting J, Nicolaides K. Blood gases in pregnancy at sea level and at high altitude. BJOG 2001; 108: 980-5.

15. Scheinfeldt LB, Tishkoff SA. Living the high life: highaltitude adaptation. Genome Biol 2010; 11: 133.

16. Postigo L, Heredia G, Illsley NP, Torricos T, Dolan C, Echalar L, et al. Where the $\mathrm{O}_{2}$ goes to: preservation of human fetal oxygen delivery and consumption at high altitude. J Physiol 2009; 587: 693-708.

17. Stuber T, Scherrer U. Circulatory adaptation to longterm high altitude exposure in Aymaras and Caucasians. Prog Cardiovasc Dis 2010; 52: 534-9.

18. Rottgardt I, Rothhammer F, Dittmar M. Native highland and lowland populations differ in $\gamma$-globin gene promoter polymorphisms related to altered fetal hemoglobin levels and delayed fetal to adult globin switch after birth. Anthropological Science 2010; 18 (1): 41-8. 